\title{
Error Bounds for Finite-Difference Approximations for a Class of Nonlinear Parabolic Systems
}

\author{
By David Hoff* and Joel Smoller**
}

\begin{abstract}
In this paper we establish error bounds for a finite-difference approximation to solutions of certain parabolic systems of the form $v_{t}+f(v)_{x}=\varepsilon v_{x x}$. We assume that the Cauchy data is of class BV, and we show that the sup norm of the error is bounded by $O(\Delta x|\ln \Delta x|)$ at positive times.
\end{abstract}

1. Introduction. In this paper we establish error bounds for a finite-difference approximation to solutions of certain parabolic systems of the form

$$
v_{t}+f(v)_{x}=\varepsilon v_{x x}, \quad(x, t) \in \mathbf{R} \times \mathbf{R}_{+},
$$

with initial data

$$
v(x, 0)=v_{0}(x), \quad x \in \mathbf{R} .
$$

Here $v \in \mathbf{R}^{n}, f \in C^{2}, \varepsilon$ is a positive constant, and $v_{0}$ is a function of bounded variation. Thus initial discontinuities are allowed, and (1.1)-(1.2) includes the classical "shock-tube" problem.

We let $x_{k}=k \Delta x$ and $t_{n}=n \Delta t$. The approximation to $v\left(x_{k}, t_{n}\right)$ is denoted by $u_{k}^{n}$, which is to be computed inductively from the finite-difference equation

$$
\frac{u_{k}^{n}-u_{k}^{n-1}}{\Delta t}=\varepsilon \frac{u_{k+1}^{n-1}-2 u_{k}^{n-1}+u_{k-1}^{n-1}}{\Delta x^{2}}-\frac{f\left(u_{k+1}^{n-1}\right)-f\left(u_{k-1}^{n-1}\right)}{2 \Delta x} .
$$

In [3], Nishida and Smoller showed that for the class of systems under consideration here, the $u_{k}^{n}$ do in fact converge to a unique, classical solution of (1.1)-(1.2). In the present paper we establish a more precise result by obtaining the error bound

$$
\sup _{k}\left|u_{k}^{n}-v\left(x_{k}, t_{n}\right)\right| \leqslant \frac{C}{\sqrt{t_{n}}}\left[\sum_{k}\left|u_{k}^{0}-v_{0}\left(x_{k}\right)\right| \Delta x+\Delta x|\ln \Delta x|\right]
$$

for $0<t_{n} \leqslant T$, where $C$ depends only on $T$, $\varepsilon$, and $f$ (Theorem 3.3 below). Actually, our exposition is essentially self-contained; indeed, with some minor modifications our arguments can be used to independently establish the existence and regularity results of [3].

Received January 20, 1984; revised May 16, 1984 and September 18, 1984.

1980 Mathematics Subject Classification. Primary 35K45, 65M15.

${ }^{*}$ Research supported in part by the NSF under Grant No. MCS-8301141.

**Research supported in part by the NSF under Grant No. MCS- 8002337. 
We also remark that computer computations performed for us by J. S. Shi indicate that the bound (1.4) is of the correct order in $\Delta x$.

We now state our assumptions concerning the system (1.1) and the mesh parameters $\Delta x$ and $\Delta t$ :

Assumption A. There is a convex set $S$ in $v$-space, in which $f^{\prime}(v)$ is bounded, which is invariant for the Lax-Friedrichs scheme

$$
u_{k}^{n}=\frac{1}{2}\left(u_{k-1}^{n-1}+u_{k+1}^{n-1}\right)+\frac{\alpha}{2}\left(f\left(u_{k-1}^{n-1}\right)-f\left(u_{k+1}^{n-1}\right)\right) .
$$

(This means that when $u_{k-1}^{n-1}$ and $u_{k+1}^{n-1}$ are in $S$, then so is the vector $u_{k}^{n}$ defined by (1.5).)

Of course, when $\alpha=\Delta t / \Delta x,(1.5)$ is a finite-difference approximation for the first-order system

$$
v_{t}+f(v)_{x}=0
$$

associated with (1.1). Now in all cases of interest, (1.6) will be strictly hyperbolic; i.e., $f^{\prime}(v)$ will have distinct, real eigenvalues $\lambda_{1}(v), \ldots, \lambda_{n}(v)$. Our precise assumption then is that $S$ is invariant for (1.5) when $\alpha$ satisfies the CFL condition

$$
\alpha=\frac{\Delta t}{\Delta x} \leqslant\left(\max _{k, u \in S}\left|\lambda_{k}(u)\right|\right)^{-1} .
$$

Assumption B. The mesh parameters $\Delta x$ and $\Delta t$ satisfy

$$
\varepsilon \Delta t / \Delta x^{2} \leqslant 1 / 2
$$

and

$$
\Delta x \leqslant 2 \varepsilon \max _{k, u \in S}\left|\lambda_{k}(u)\right| .
$$

These mesh conditions insure that $S$ is invariant for our difference scheme (1.3). To see this, note that if $\beta=\Delta t / \Delta x^{2}$, then (1.3) may be written in the form

(1.9) $u_{k}^{n}=(1-2 \varepsilon \beta) u_{k}^{n-1}+2 \varepsilon \beta\left[\frac{1}{2}\left(u_{k-1}^{n-1}+u_{k+1}^{n-1}\right)+\frac{\Delta x}{4 \varepsilon}\left(f\left(u_{k-1}^{n-1}\right)-f\left(u_{k+1}^{n-1}\right)\right)\right]$.

Thus if $u_{k+1}^{n-1}$ are points of $S$, then so is the term in the above brackets, in view of Assumption A and (1.8). Furthermore, since $1-2 \varepsilon \beta \geqslant 0$ by (1.7), (1.9) shows that $u_{k}^{n}$ is a convex combination of points in the convex set $S$.

Examples of hyperbolic systems (1.6) which satisfy Assumption A are scalar equations, the " $p$-system"

$$
u_{t}+p(v)_{x}=0, \quad v_{t}-u_{x}=0 \quad\left(p^{\prime}(v)<0<p^{\prime \prime}(v)\right)
$$

(see Hoff [1] for details concerning the invariant regions for (1.5) for this example), and those $2 \times 2$ systems whose shock and rarefaction curves coincide (see Temple [5]). A complete discussion of invariant regions for weak solutions of (1.6) is given in Hoff [2]. When (1.6) is genuinely nonlinear, such invariant regions are necessarily convex (but not otherwise), and in this case they are always invariant for the Lax-Friedrichs scheme (1.5), at least when $u_{k-1}^{n-1}$ and $u_{k+1}^{n-1}$ are sufficiently close.

We remark that, although different error bounds could be anticipated for smoother initial data, we have decided to focus attention on initial data of bounded variation because the system (1.1) is of interest primarily as a perturbation of the corresponding system of conservation laws (Eq. (1.1) with $\varepsilon=0$ ), and the class BV is the 
natural space for data and solutions of these conservation laws; see Glimm [6]. Moreover, there is now renewed interest (DiPerna [7]) in the problem of comparing solutions of the viscous equation (1.1) with the solution of the corresponding system of conservation laws. This question presupposes of course, that one can actually solve the system (1.1) globally for initial data in BV. The existence portion of our results gives an affirmative answer for a large class of systems (1.1) of interest.

Because our goal is to establish an error bound in sup norm, even when the initial data (1.2) is discontinuous, we shall need to exploit the regularizing properties of a certain discrete heat operator. Now, since this operator is orthogonally diagonalizable, one could easily show that, for positive times, it maps the Sobolev spaces $H^{s}$ continuously into $H^{s+p}$ for $p>0$. On the other hand, the corresponding regularizing properties on the space BV are less well-known. We therefore give a complete, self-contained exposition of these facts in Section 2. Then in Section 3 we apply these results to obtain the error bound (1.4) for the scheme (1.3).

We shall use the following notation conventions throughout the paper. If $u=$ $\left(\ldots, u_{k}, \ldots\right)$ is an infinite vector, we let

$$
|u|_{1}=\sum_{k}\left|u_{k}\right| \Delta x \text { and }|u|_{\infty}=\sup _{k}\left|u_{k}\right| .
$$

Also, for functions $v=v(x),\|v\|_{p}$ will be the usual $L^{p}$-norm, $1 \leqslant p \leqslant \infty$.

We let $K$ be the usual heat kernel

$$
K(x, t)=(1 / \sqrt{4 \pi \varepsilon t}) e^{-x^{2} / \sqrt{4 \varepsilon t}} .
$$

The solution $v(t)=v(\cdot, t)$ of (1.1)-(1.2) then satisfies the standard representation

$$
v(t)=K(t) * v_{0}-\int_{0}^{t} K_{x}(t-s) * f(v(s)) d s
$$

see [4].

2. The Discrete Heat Operator. In this section we study the standard explicit method for the scalar heat equation

$$
v_{t}=\varepsilon v_{x x}
$$

in the case that the initial data $v(x, 0)$ has finite total variation. Thus $v\left(x_{k}, t_{n}\right)$ is approximated by $u_{k}^{n}$, which is computed from the finite-difference scheme

$$
\frac{u_{k}^{n}-u_{k}^{n-1}}{\Delta t}=\varepsilon \frac{u_{k+1}^{n-1}-2 u_{k}^{n-1}+u_{k-1}^{n-1}}{\Delta x^{2}} .
$$

Using the notations

$$
\delta^{2} u_{k}=u_{k+1}-2 u_{k}+u_{k-1}, \quad u^{n}=\left(\ldots, u_{k}^{n}, \ldots\right),
$$

and, as before,

$$
\beta=\Delta t / \Delta x^{2},
$$

we may rewrite the scheme (2.1) in the form

$$
u^{n}=\left(I+\varepsilon \beta \delta^{2}\right) u^{n-1} .
$$

We dofine below a discrete fundamental solution for (2.2); we investigate its properties in Theorem 2.2; and in Theorem 2.3 we derive a bound for the error

$$
\sup _{k}\left|u_{k}^{n}-v\left(x_{k}, t_{n}\right)\right| \text {. }
$$


Definitions. The fundamental solution for (2.2) is the sequence $a_{k}^{n}$ defined by

$$
a_{k}^{0}= \begin{cases}1 / \Delta x, & k=0, \\ 0, & k \neq 0,\end{cases}
$$

and

$$
a^{n}=\left(I+\varepsilon \beta \delta^{2}\right)^{n} a^{0} .
$$

Also, given sequences $w_{k}$ and $z_{k}$, one of which has finite support, we define the discrete convolution by

$$
(w * z)_{k}=\sum_{j} w_{k-j} z_{j} \Delta x .
$$

It is clear that the usual algebraic properties of convolution hold, and that if $\delta_{+}$is the forward-difference operator, $\delta_{+} w_{k}=w_{k+1}-w_{k}$, then

$$
\delta_{+}(w * z)=\left(\delta_{+} w\right) * z=w *\left(\delta_{+} z\right)
$$

Finally, we have the inequalities

$$
|w * z|_{1} \leqslant|w|_{1}|z|_{1} \text { and }|w * z|_{\infty} \leqslant|w|_{1}|z|_{\infty} .
$$

In the following theorem we derive the discrete version of Duhamel's theorem for the heat equation. This result will enable us later on to give a representation of the solution of the difference equation (1.3) in terms of the fundamental solution $a_{k}^{n}$.

THEOREM 2.1. The solution $u_{k}^{n}$ of the nonhomogeneous difference equation

$$
\frac{u_{k}^{n}-u_{k}^{n-1}}{\Delta t}=\varepsilon \frac{\delta^{2} u_{k}^{n-1}}{\Delta x^{2}}+f_{k}^{n-1}
$$

is given by

$$
u^{n}=a^{n} * u^{0}+\sum_{j=1}^{n} a^{n-j} * f^{j-1} \Delta t
$$

Proof. We have that

$$
\begin{aligned}
u^{n-u^{n-1}} & =\left(a^{n}-a^{n-1}\right) * u^{0}+a^{0} * f^{n-1} \Delta t+\sum_{j=1}^{n-1}\left(a^{n-j}-a^{n-1-j}\right) * f^{j-1} \Delta t \\
& =\varepsilon \beta \delta^{2} a^{n-1} * u^{0}+f^{n-1} \Delta t+\sum_{j=1}^{n-1} \varepsilon \beta \delta^{2} a^{n-1-j} * f^{j-1} \Delta t \\
& =\varepsilon \beta \delta^{2}\left(a^{n-1} * u^{0}+\sum_{j=1}^{n-1} a^{n-1-j} * f^{j-1} \Delta t\right)+f^{n-1} \Delta t \\
& =\varepsilon \beta \delta^{2} u^{n-1}+f^{n-1} \Delta t .
\end{aligned}
$$

Next we collect together various facts about the fundamental solution $a_{k}^{n}$ which will be required in the subsequent analysis.

THEOREM 2.2. Assume that $\varepsilon \beta<1 / 2$. Then the sequence $a_{k}^{n}$ satisfies

$$
\begin{gathered}
\sum_{k} a_{k}^{n}=1, \\
0 \leqslant a_{k}^{n} \leqslant C_{1} / \sqrt{t_{n}},
\end{gathered}
$$


and

$$
\left|\delta_{+}^{p} a_{k}^{n} / \Delta x^{p}\right|_{1} \leqslant C_{2} / t_{n}^{p / 2} .
$$

Here $C_{1}$ is a constant which depends only on $\varepsilon$, and $C_{2}$ depends on $\varepsilon$ and $p$.

Proof. (2.3) holds for $n=0$ by construction, and the general case follows by induction on $n$, since

$$
\sum_{k}\left(a_{k}^{n}-a_{k}^{n-1}\right)=\varepsilon \beta \sum_{k} \delta^{2} a_{k}^{n-1}=0 .
$$

Similarly, the lower bound in (2.4) holds for $n=0$ by construction, and the general case follows by induction, since the difference equation (2.1) shows that $a_{k}^{n}$ is a convex combination of $a_{k}^{n-1}$ and $a_{k \pm 1}^{n-1}$.

To prove the upper bound in (2.4) we let $w^{n}(\theta)$ be the trigonometric polynomial

$$
w^{n}(\theta)=\sum_{k} a_{k}^{n} e^{i k \theta} \text {. }
$$

Applying the difference equation (2.1), we find that

$$
w^{n}(\theta)=\gamma(\theta) w^{n-1}(\theta),
$$

where

$$
\gamma(\theta)=1+2 \varepsilon \beta(\cos \theta-1) .
$$

Thus

$$
w^{n}(\theta)=\gamma(\theta)^{n} w^{0}(\theta)=(1 / \Delta x) \gamma(\theta)^{n} .
$$

Now, it is easy to see that our hypothesis on $\beta$ implies that

$$
|\gamma(\theta)| \leqslant e^{-c \theta^{2}}
$$

for some positive constant $C$ when $|\theta| \leqslant \pi$. Therefore,

$$
\begin{aligned}
a_{k}^{n} & =\frac{1}{2 \pi} \int_{-\pi}^{\pi} e^{-i k \theta} w^{n}(\theta) d \theta<\frac{1}{2 \pi \Delta x} \int_{-\pi}^{\pi} e^{-C n \theta^{2}} d \theta \\
& \leqslant \frac{1}{2 \pi n^{1 / 2} \Delta x} \int_{-\infty}^{\infty} e^{-C y^{2}} d y=\frac{C}{\sqrt{t_{n}}} .
\end{aligned}
$$

We prove (2.5) first for the case that $p=1$. Let

$$
z^{n}(\theta)=\sum_{k}\left(\delta_{+} a_{k}^{n}\right) e^{i k \theta} .
$$

Again, using the difference equation (2.1), we find that

$$
z^{n}(\theta)=\left(e^{-i \theta}-1\right) \sum_{k} a_{k}^{n} e^{i k \theta}=\left(\frac{e^{-i \theta}-1}{\Delta x}\right) \gamma(\theta)^{n} .
$$

Next, define

$$
\tilde{z}^{n}(\theta)=\sum_{k}\left|\delta_{+} a_{k}^{n}\right| e^{i k \theta}
$$

Observe that

$$
\tilde{z}^{n}(\pi)=\sum_{k}(-1)^{k}\left|\delta_{+} a_{k}^{n}\right|=0,
$$


because of the obvious symmetry $a_{k}^{n}=a_{-k}^{n}$. Therefore,

$$
\begin{aligned}
\sum_{k}\left|\frac{\delta_{+} a_{k}^{n}}{\Delta x}\right| \Delta x & =\tilde{z}^{n}(0)=\left[\int_{0}^{\pi} \frac{d}{d \theta} \tilde{z}^{n}(\theta)^{2} d \theta\right]^{1 / 2} \\
& \leqslant C\left\|\tilde{z}^{n}\right\|_{L^{2}}^{1 / 2}\left\|\frac{d \tilde{z}^{n}}{d \theta}\right\|_{L^{2}}^{1 / 2}=C\left\|z^{n}\right\|_{L^{2}}^{1 / 2}\left\|\frac{d z^{n}}{d \theta}\right\|_{L^{2}}^{1 / 2} .
\end{aligned}
$$

However, (2.6) and (2.7) show that

so that

$$
\left|z^{n}(\theta)\right| \leqslant(C|\theta| / \Delta x) e^{-C n \theta^{2}},
$$

$$
\left\|z^{n}\right\|_{L^{2}} \leqslant C / n^{3 / 4} \Delta x
$$

Similarly,

$$
\left|\frac{d z^{n}}{d \theta}(\theta)\right| \leqslant \frac{C}{\Delta x}\left(1+n \theta^{2}\right) e^{-C n \theta^{2}}
$$

so that

$$
\left\|\frac{d z^{n}}{d \theta}\right\|_{L^{2}} \leqslant \frac{C}{n^{1 / 4} \Delta x} .
$$

Substituting these estimates into (2.8), we then have

$$
\left|\frac{\delta_{+} a_{k}^{n}}{\Delta x}\right|_{1} \leqslant C / n^{1 / 2} \Delta x=C / \sqrt{t_{n}},
$$

as required.

For general $p$ we let $m$ be the greatest integer in $n / p$ and $n=m(p-1)+q$. It is easy to see that there is an integer $N=N(p)$ such that, when $n \geqslant N(p)$, the integers $m$ and $q$ satisfy $m, q \geqslant n / 2 p$. For $\operatorname{such} n$, then,

$$
\begin{aligned}
\left|\frac{\delta_{+}^{p} a^{n}}{\Delta x^{p}}\right|_{1} & =\mid \underbrace{\frac{\delta_{+} a^{m}}{\Delta x} * \cdots * \frac{\delta_{+} a^{m}}{\Delta x}}_{p-1 \text { factors }})\left.* \frac{\delta_{+} a^{q}}{\Delta x}\right|_{1} \\
& \leqslant\left|\frac{\delta_{+} a^{m}}{\Delta x}\right|_{1}^{p-1}\left|\frac{\delta_{+} a^{q}}{\Delta x}\right|_{1} \leqslant \frac{C}{t_{m}^{(p-1) / 2}} \cdot \frac{C}{t_{q}^{1 / 2}} \leqslant \frac{C(\varepsilon, p)}{t_{n}^{p / 2}} .
\end{aligned}
$$

And when $n \leqslant N(p)$

$$
\left|\frac{\delta_{+}^{p} a_{k}^{n}}{\Delta x^{p}}\right| \leqslant \frac{\left|a^{n}\right|_{\infty}}{\Delta x^{p}} \leqslant \frac{C}{\Delta x^{p} t_{n}^{1 / 2}},
$$

so that

$$
\begin{aligned}
\left|\frac{\delta_{+}^{p} a^{n}}{\Delta x^{p}}\right|_{1} & \leqslant \sum_{k=-N(p)}^{N(p)} \frac{C \Delta x}{\Delta x^{p} t_{n}^{1 / 2}}=\frac{C}{\Delta t^{(p-1) / 2} t_{n}^{1 / 2}} \\
& =\frac{C n^{(p-1) / 2}}{t_{n}^{p / 2}} \leqslant \frac{C(\varepsilon, p)}{t_{n}^{p / 2}} \cdot \square
\end{aligned}
$$

Next we establish an $L^{\infty}$-error bound for the approximate solution of the discrete heat equation in the case that the initial data $v$ has finite total variation. This result will be crucial for the derivation of error bounds for the approximate solutions of the regularized conservation laws (1.1). 
In the following, $K$ will be the usual heat kernel defined in (1.10). Thus the exact solution of the heat equation at time $t$ is $v(t)=K(t) * v$. Also, $P$ will denote the operator which projects functions of $x$ onto vectors by pointwise evaluation, that is,

$$
(P w)_{k}=w\left(x_{k}\right)
$$

For example, if $u^{n}$ is the solution of the finite-difference equation (2.1) with initial data $u_{k}^{0}=v\left(x_{k}\right)$, then $u^{n}=a^{n} * P v$.

Theorem 2.3. Let $v \in \mathrm{BV}$, and let $V=$ Tot.Var.( $(v)$. Then, given $T>0$, the bound

$$
\left|a^{n} * P v-P\left(K\left(t_{n}\right) * v\right)\right|_{\infty} \leqslant C(T) \frac{V}{\sqrt{t_{n}}} \Delta x|\ln \Delta x|
$$

is valid for $0<t_{n} \leqslant T$.

Proof. We adopt the following notations:

$$
v^{n}=P\left(K\left(t_{n}\right) * v\right) \text { and } u^{n}=a^{n} *(P v) .
$$

In addition, if $j_{h}$ is the standard mollifier with support in $[-h, h]$, then

$$
v_{h}=j_{h} * v, \quad v_{h}^{n}=P\left(K\left(t_{n}\right) * v_{h}\right), \quad \text { and } \quad u_{h}^{n}=a^{n} *\left(P v_{h}\right) .
$$

We then have

$$
\begin{aligned}
\left|a^{n} * P v-P\left(K\left(t_{n}\right) * v\right)\right|_{\infty} & =\left|u^{n}-v^{n}\right|_{\infty} \\
& \leqslant\left|u^{n}-u_{h}^{n}\right|_{\infty}+\left|u_{h}^{n}-v_{h}^{n}\right|_{\infty}+\left|v_{h}^{n}-v^{n}\right|_{\infty} .
\end{aligned}
$$

The proof consists of showing that when $h=\Delta x$, each of the three terms on the right side of (2.10) is bounded by the right side of (2.9).

The third term on the right of $(2.10)$ is bounded by

$$
\left\|K\left(t_{n}\right) *\left(v_{h}-v\right)\right\|_{\infty} \leqslant\left\|K\left(t_{n}\right)\right\|_{\infty}\left\|v_{h}-v\right\|_{1} \leqslant \frac{C}{\sqrt{t_{n}}}\left\|v_{h}-v\right\|_{1} .
$$

But for smooth functions $v(x)$,

$$
\begin{aligned}
\left|v_{h}(x)-v(x)\right| & \leqslant \int j_{h}(x-y)|v(y)-v(x)| d y \\
& \leqslant \int_{-\infty}^{\infty} \int_{x-h}^{x+h} j_{h}(x-y)\left|v^{\prime}(s)\right| d s d y=\int_{x-y}^{x+h}\left|v^{\prime}(s)\right| d s,
\end{aligned}
$$

so that

$$
\begin{aligned}
\left\|v_{h}-v\right\|_{1} & \leqslant \int_{-\infty}^{\infty} \int_{x-h}^{x+h}\left|v^{\prime}(s)\right| d s d x \\
& =\int_{-\infty}^{\infty} \int_{s-h}^{s+h}\left|v^{\prime}(s)\right| d x d s=2 h V .
\end{aligned}
$$

The same result holds for $v \in \mathrm{BV}$ by a simple approximation argument. Thus, when $h=\Delta x$,

$$
\left|v_{h}^{n}-v^{n}\right|_{\infty} \leqslant\left(C V / \sqrt{t_{n}}\right) \Delta x .
$$

The bound for the first term on the right of $(2.10)$ is similar:

$$
\begin{aligned}
\left|u^{n}-u_{h}^{n}\right|_{\infty} & =\left|a^{n} * P\left(v-v_{h}\right)\right|_{\infty} \leqslant\left|a^{n}\right|_{\infty}\left|P\left(v-v_{h}\right)\right|_{1} \\
& \leqslant\left(C / \sqrt{t_{n}}\right)\left|P\left(v-v_{h}\right)\right|_{1},
\end{aligned}
$$


by Theorem 2.2. And as above,

$$
\begin{aligned}
\left|v\left(x_{k}\right)-v_{h}\left(x_{k}\right)\right| & \leqslant \int_{x_{k}-h}^{x_{k}+h}\left|v\left(x_{k}\right)-v(x)\right| j_{h}\left(x_{k}-x\right) d x \\
& \leqslant \operatorname{Var}\left[v ;\left(x_{k}-h, x_{k}+h\right)\right]
\end{aligned}
$$

so that

$$
\begin{aligned}
\left|P\left(v-v_{h}\right)\right|_{1} & =\sum_{k}\left|v\left(x_{k}\right)-v_{h}\left(x_{k}\right)\right| \Delta x \\
& \leqslant \sum_{k} \operatorname{Var}\left[v ;\left(x_{k}-h, x_{k}+h\right)\right] \Delta x=2 V \Delta x
\end{aligned}
$$

when $h=\Delta x$. Thus

$$
\left|u^{n}-u_{h}^{n}\right|_{\infty} \leqslant\left(C V / \sqrt{t_{n}}\right) \Delta x .
$$

To estimate the second term on the right of (2.10) we define the vector $\sigma^{n-1}$ by

$$
\frac{v_{h}^{n}-v_{h}^{n-1}}{\Delta t}=\varepsilon \frac{\delta^{2} v_{h}^{n-1}}{\Delta x^{2}}+\sigma^{n-1} \text {. }
$$

We shall show below that, when $h=\Delta x$,

$$
\left|\sigma^{n}\right|_{1} \leqslant C V \begin{cases}\Delta x^{-1}, & n \geqslant 0 \\ t_{n}^{-1 / 2}, & n>0 \\ \Delta x t_{n}^{-1}, & n>0\end{cases}
$$

Now, by Theorem 2.1,

$$
v_{h}^{n}=a^{n} * P v_{h}+\sum_{j=1}^{n-1} a^{n-j} * \sigma^{j-1} \Delta t
$$

and, by definition,

$$
u_{h}^{n}=a^{n} * P v_{h}
$$

Thus,

$$
\begin{aligned}
v_{h}^{n}-u_{h}^{n} & =\sum_{j=1}^{n} a^{n-j} * \sigma^{j-1} \Delta t \\
& =a^{n-1} * \sigma^{0} \Delta t+\sum_{j=2}^{n-1} a^{n-j} * \sigma^{j-1} \Delta t+a^{0} * \sigma^{n-1} \Delta t
\end{aligned}
$$

and

$$
\left|v_{h}^{n}-u_{h}^{n}\right|_{\infty} \leqslant \frac{C}{\sqrt{t_{n-1}}}\left|\sigma^{0}\right|_{1} \Delta t+C \sum_{j=2}^{n-1} \frac{\left|\sigma^{j-1}\right|_{1} \Delta t}{\sqrt{t_{n}-t_{j}}}+\left|\sigma^{n-1}\right|_{1} \frac{\Delta t}{\Delta x} .
$$

Using each of the estimates (2.11), we obtain

$$
\left|v_{h}^{n}-u_{h}^{n}\right|_{\infty} \leqslant C V\left[\frac{1}{\sqrt{t_{n-1}}} \frac{\Delta t}{\Delta x}+\sum_{j=2}^{n-1} \frac{\Delta x \Delta t}{\sqrt{t_{n}-t_{j}} t_{j-1}}\right] .
$$

Now the result (2.9) is trivial if $t_{n}=O(\Delta t)$, and for $t_{n} \geqslant 2 \Delta t$ we have that

$$
1 / \sqrt{t_{n-1}} \leqslant \sqrt{2} / \sqrt{t_{n}} \text {. }
$$


Since $\Delta t / \Delta x=O(\Delta x)$, we may conclude that

$$
\left|v_{h}^{n}-u_{h}^{n}\right|_{\infty} \leqslant C V\left[\frac{\Delta x}{\sqrt{t_{n}}}+\Delta x \sum_{j=2}^{n-1} \frac{\Delta t}{\sqrt{t_{n}-t_{j}} t_{j-1}}\right] .
$$

The sum here is

$$
\sum_{j=2}^{n-1} \frac{j}{j-1} \frac{\Delta t}{\sqrt{t_{n}-t_{j}} t_{j}} \leqslant 2 \int_{\Delta t}^{t_{n}} \frac{d s}{\sqrt{t_{n}-s} s}=\frac{2}{\sqrt{t_{n}}} \int_{\Delta t / t_{n}}^{1} \frac{d y}{\sqrt{1-y} y} .
$$

Now since the function $\sqrt{1-y}+y$ is bounded away from 0 on $[0,1]$, we have that

$$
\frac{1}{\sqrt{1-y} y} \leqslant C\left[\frac{1}{y}+\frac{1}{\sqrt{1-y}}\right] .
$$

Therefore the sum in (2.12) is bounded by

$$
\begin{aligned}
\frac{C}{\sqrt{t_{n}}} \int_{\Delta t / t_{n}}^{1}\left[\frac{1}{y}+\frac{1}{\sqrt{1-y}}\right] d y & =C\left[\ln \left(\frac{t_{n}}{\Delta t}\right)+\sqrt{1-\frac{\Delta t}{t_{n}}}\right] \\
& \leqslant C(T)(1+|\ln \Delta x|),
\end{aligned}
$$

and (2.12) shows that

$$
\left|v_{h}^{n}-u_{h}^{n}\right| \leqslant C(T)\left(1+1 / \sqrt{t_{n}}\right) V \Delta x|\ln \Delta x|,
$$

as required.

We still have to prove (2.11). From its definition we have that

$$
\left|\sigma^{n}\right|_{1} \leqslant\left|\frac{v_{h}^{n+1}-v_{h}^{n}}{\Delta t}-P \frac{\partial v_{h}}{\partial t}\left(t_{n}\right)\right|_{1}+\varepsilon\left|\frac{\delta^{2} v_{h}^{n}}{\Delta x^{2}}-P \frac{\partial^{2} v_{h}\left(t_{n}\right)}{\partial x^{2}}\right|_{1}
$$

We shall estimate only the first term here. The argument for the second term is somewhat easier. First, note the simple fact that if $w$ is a smooth integrable function of $x$,

$$
\left|\sum_{k} w_{k} \Delta x-\int w d x\right| \leqslant \Delta x \int\left|w^{\prime}(x)\right| d x .
$$

The term in question is then estimated as follows:

$$
\begin{aligned}
\sum_{k} \mid & \frac{v_{h}\left(x_{k}, t_{n+1}\right)-v_{h}\left(x_{k}, t_{n}\right)}{\Delta t}-\frac{\partial v_{h}}{\partial t}\left(x_{k}, t_{n}\right) \mid \Delta x \\
& \leqslant \sum_{k} \frac{\Delta x}{\Delta t} \int_{t_{n}}^{t_{n+1}}\left(t_{n+1}-s\right)\left|\frac{\partial^{2} v_{h}}{\partial t^{2}}\left(x_{k}, s\right)\right| d s \\
& =\frac{1}{\Delta t} \int_{t_{n}}^{t_{n+1}}\left(t_{n+1}-s\right)\left[\sum_{k}\left|\frac{\partial^{2} v_{h}}{\partial t^{2}}\left(x_{k}, s\right)\right| \Delta x\right] d s \\
& \leqslant \frac{1}{\Delta t} \int_{t_{n}}^{t_{n+1}}\left(t_{n+1}-s\right)\left[\int\left|\frac{\partial^{2} v_{h}}{\partial t^{2}}(x, s)\right| d x+\Delta x \int\left|\frac{\partial^{3} v_{h}}{\partial t^{2} \partial x}(x, s)\right| d x\right] d s \\
& \leqslant C \Delta t \sup _{t_{n} \leqslant s \leqslant t_{n+1}}\left[\left\|D_{x}^{4} v_{h}(s)\right\|_{1}+\Delta x\left\|D_{x}^{5} v_{h}(s)\right\|_{1}\right],
\end{aligned}
$$


since $v_{h}$ satisfies the heat equation. But

$$
v_{h}(s)=K(s) * j_{h} * v,
$$

so that if $p$ and $q$ are nonnegative integers with $p+q=3$,

$$
\begin{aligned}
\left\|D_{x}^{4} v_{h}(s)\right\|_{1} & \leqslant\left\|D_{x}^{p} K(s)\right\|_{1}\left\|D_{x}^{q} j_{h}\right\|_{1}\left\|D_{x} v\right\|_{1} \\
& \leqslant C V s^{-p / 2} h^{-q} \leqslant C V s^{-p / 2} \Delta x^{-q}
\end{aligned}
$$

when $h=\Delta x$. Similarly,

$$
\Delta x\left\|D_{x}^{5} v_{h}(s)\right\|_{1} \leqslant \Delta x\left\|D_{x}^{p} K(s)\right\|_{1}\left\|D_{x}^{q+1} j_{h}\right\|_{1}\left\|D_{x} v\right\|_{1} \leqslant C V s^{-p / 2} \Delta x^{-(q+1)} .
$$

Substituting these bounds into (2.14), we thus find that the first term on the right of $(2.13)$ is bounded by

$$
C V t_{n}^{-p / 2} \Delta t \Delta x^{-q}=C V t_{n}^{-p / 2} \Delta x^{p-1}
$$

for $p=0,1,2$. The estimate for the other term in (2.13) is similar.

3. Proof of the Error Bound. In this section we apply the results of Section 2 to prove the error bound (1.4) for the approximate solutions $u_{k}^{n}$ of the difference scheme (1.3). We assume throughout this section that Assumptions A and B of Section 1 hold. Therefore, as remarked in the introduction, the convex set $S$ is invariant for the scheme (1.3), and the results of [3] then apply to show that we have the bounds

$$
\left|u_{k}^{n}\right|, \sum_{k}\left|u_{k+1}^{n}-u_{k}^{n}\right| \leqslant C(T), \quad 0 \leqslant t_{n} \leqslant T .
$$

Moreover, the $u_{k}^{n}$ converge to the unique classical solution $v(x, t)$ of (1.1)-(1.2), which therefore satisfies $v(x, t) \in S$ and

$$
\|v(\cdot, t)\|_{\infty},\left\|v_{x}(\cdot, t)\right\|_{1} \leqslant C(T) V, \quad 0 \leqslant t \leqslant T .
$$

(We remind the reader that, as in Section $1, u$ and $v$ are vectors in $\mathbf{R}^{n}$.)

We begin by estimating the derivatives of the exact solution $v(x, t)$.

LEMMA 3.1. Given $T>0$ there is a constant $C$ depending on $T, \varepsilon$, and $f$ such that the solution $v(x, t)$ of $(1.1)-(1.2)$ satisfies

$$
\left\|v_{x}(t)\right\|_{\infty} \leqslant C V / \sqrt{t}
$$

and

$$
\left\|v_{x x}(t)\right\|_{1} \leqslant C\left(V+V^{2}\right) / \sqrt{t}
$$

for $t \leqslant T$. Here $V$ is the total variation of $v_{0}$.

Proof. Using the bound $\left\|K_{x}(t)\right\|_{1} \leqslant C / \sqrt{t}$ in the representation (1.11), we obtain

$$
\left\|v_{x}(t)\right\|_{\infty} \leqslant \frac{C V}{\sqrt{t}}+C \int_{0}^{t} \frac{\left\|v_{x}(s)\right\|_{\infty}}{\sqrt{t-s}} d s .
$$

Now let $a(t)=\sqrt{t}\left\|v_{x}(t)\right\|_{\infty}$. Multiplying (3.3) by $\sqrt{t}$, we then obtain that

$$
a(t) \leqslant C V+C \sqrt{t} \int_{0}^{t} \frac{a(s) d s}{\sqrt{s(t-s)}} .
$$


Substituting this estimate back into the integral in (3.4), we then obtain

$$
\begin{aligned}
a(t) & \leqslant C V+C \sqrt{t} \int_{0}^{t} \frac{C V d s}{\sqrt{s(t-s)}}+C \sqrt{t} \int_{0}^{t} \frac{C \sqrt{s} \int_{0}^{s}(a(\tau) d \tau) / \sqrt{\tau(s-\tau)}}{\sqrt{s(t-s)}} d s \\
& =C(T) V+C(T) \int_{0}^{t} \frac{a(\tau)}{\sqrt{\tau}}\left(\int_{\tau}^{t} \frac{d s}{\sqrt{(t-s)(s-\tau)}}\right) d \tau .
\end{aligned}
$$

A simple change of variables shows that the inner integral here is a constant. It therefore follows from Gronwall's inequality that

$$
a(t) \leqslant C(T) V,
$$

which is (3.1).

The proof of (3.2) is similar. From the representation (1.11) we have that

$$
v_{x x}(t)=K_{x}(t-s) * v_{0}^{\prime}-\int_{0}^{t} K_{x}(t-s) * f(v(s))_{x x} d s,
$$

so that

$$
\left\|v_{x x}(t)\right\|_{1} \leqslant \frac{C V}{\sqrt{t}}+C \int_{0}^{t} \frac{\left\|v_{x x}(s)\right\|_{1}+\left\|v_{x}(s)\right\|_{\infty}\left\|v_{x}(s)\right\|_{1}}{\sqrt{t-s}} d s .
$$

The second term in the integral is bounded by

$$
C \int_{0}^{t} \frac{V^{2} d s}{\sqrt{s(t-s)}}=C V^{2}
$$

by (3.1). Thus,

$$
\left\|v_{x x}(t)\right\|_{1} \leqslant C\left(\frac{V}{\sqrt{t}}+V^{2}\right)+C \int_{0}^{t} \frac{\left\|v_{x x}(s)\right\|_{1} d s}{\sqrt{t-s}} .
$$

This is nearly identical in form to (3.3); and so (3.2) follows as before. The proof is complete.

As an immediate corollary to (3.1) and (3.2) we observe that

$$
\left\|f(v(t))_{x x}\right\|_{1},\left\|v_{t}(t)\right\|_{1} \leqslant C\left(\frac{V+V^{2}}{\sqrt{t}}\right), \quad 0<t \leqslant T .
$$

As in Section 2, $P$ will denote the projection operator, defined for a given vector function $w$ of $x$, by

$$
P w=\left(\ldots, w\left(x_{k}\right), w\left(x_{k+1}\right), \ldots\right)
$$

and if $v$ is the exact solution of $(1.1)$, the vector $P v\left(\cdot, t_{n}\right)$ will be denoted by $v^{n}$. The truncation error associated with $v$ is then the vector $\tau^{n}$ defined by

$$
v^{n}=a^{n} * P v_{0}-\sum_{j=1}^{n} a^{n-j} * \frac{\delta}{2 \Delta x} P f\left(v\left(t_{j-1}\right)\right) \Delta t+\tau^{n} .
$$

We then have the following bound for $\tau^{n}$.

Lemma 3.2. Given $T>0$ there is a constant $C$, depending on $T, \varepsilon$, and $f$, such that the bound

$$
\left|\tau^{n}\right|_{\infty} \leqslant C\left(\left(V+V^{2}\right) / \sqrt{t_{n}}\right) \Delta x|\ln \Delta x|
$$

holds for $0<t_{n} \leqslant T$. 
Proof. From the representation (1.11) we have that

$$
v^{n}=P\left[K\left(t_{n}\right) * v_{0}\right]-\int_{0}^{t_{n}} P\left[K\left(t_{n}-s\right) * f(v(s))_{x}\right] d s .
$$

Subtracting this from the definition of $\tau^{n}$, (3.6), we then have that

$$
\begin{aligned}
\tau^{n}= & \left\{P\left[K\left(t_{n}\right) * v_{0}\right]-a^{n} * P v_{0}\right\} \\
& +\sum_{j=1}^{n} a^{n-j} *\left[\frac{\delta}{2 \Delta x} P f\left(v\left(t_{j-1}\right)\right)-P \frac{\partial}{\partial x} f\left(v\left(t_{j-1}\right)\right)\right] \Delta t \\
& +\sum_{j=1}^{n}\left\{a^{n-j} * P \frac{\partial}{\partial x} f\left(v\left(t_{j-1}\right)\right)-P\left[K\left(t_{n}-t_{j}\right) * \frac{\partial}{\partial x} f\left(v\left(t_{j-1}\right)\right)\right]\right\} \Delta t \\
& +P\left[\sum_{j=1}^{n} K\left(t_{n}-t_{j}\right) * \frac{\partial}{\partial x} f\left(v\left(t_{j-1}\right)\right) \Delta t\right. \\
& \left.\quad-\int_{0}^{t_{n}} K\left(t_{n}-s\right) * \frac{\partial}{\partial x} f(v(s)) d s\right] \\
\equiv & \text { I }+ \text { II }+ \text { III }+ \text { IV. }
\end{aligned}
$$

We shall show that each of the terms I-IV is bounded (in the $|\cdot|_{\infty}$ norm) by the right side of (3.7). The bound for I is immediate from Theorem 2.3. For term II, note first that the $j=1$ and $j=n$ summands are bounded by

$$
\left\|f\left(v\left(t_{j-1}\right)\right)\right\|_{\infty}(\Delta t / \Delta x) .
$$

Now $\Delta t / \Delta x=O(\Delta x)$, and it is easy to see that

$$
\|f(v(t))\|_{\infty} \leqslant C \text { T.V. }(v(t)) \leqslant C(T) V .
$$

(This requires the normalization $f\left(v_{0}(-\infty)\right)=0$, which entails no loss of generality.) Thus the $j=1$ and $j=n$ terms in II are bounded by $C V \Delta x$ as required. Next observe that

$$
\left|\frac{f\left(v\left(x_{k+1}, t\right)\right)-f\left(v\left(x_{k-1}, t\right)\right)}{2 \Delta x}-\frac{\partial}{\partial x} f\left(v\left(x_{k}, t\right)\right)\right| \leqslant \int_{x_{k-1}}^{x_{k+1}}\left|\frac{\partial^{2}}{\partial x^{2}} f(v(x, t))\right| d x,
$$

so that

$$
\begin{aligned}
& \left|P \frac{\partial}{\partial x} f\left(v\left(t_{j-1}\right)\right)-\frac{\delta}{2 \Delta x} P f\left(v\left(t_{j-1}\right)\right)\right|_{1} \\
& \quad \leqslant 2 \Delta x\left\|\frac{\partial^{2}}{\partial x^{2}} f\left(v\left(t_{j-1}\right)\right)\right\|_{1} \leqslant C\left(\frac{V+V^{2}}{\sqrt{t_{j-1}}}\right) \Delta x
\end{aligned}
$$

by Lemma 3.1. Thus the sup-norm of the remaining terms in II is bounded by

$$
\sum_{j=2}^{n-1} \frac{C}{\sqrt{t_{n}-t_{j}}}\left(\frac{V+V^{2}}{\sqrt{t_{j-1}}}\right) \Delta x \Delta t \leqslant C\left(V^{2}+V\right) \Delta x
$$

as required. 
In term III the $j=n$ term is 0 and the $j=1$ term may be discarded as before. Applying Theorem 2.3 and Lemma 3.1, we find that the remaining terms are bounded by

$$
\begin{aligned}
& C \sum_{j=2}^{n-1} \frac{1}{\sqrt{t_{n}-t_{j-1}}}\left\|\frac{\partial^{2}}{\partial x^{2}} f\left(v\left(t_{j-1}\right)\right)\right\|_{1} \Delta x|\ln \Delta x| \Delta t \\
& \leqslant C \sum_{j=2}^{n-1} \frac{1}{\sqrt{t_{n}-t_{j-1}}}\left(\frac{V+V^{2}}{\sqrt{t_{j-1}}}\right) \Delta x|\ln \Delta x| \Delta t \\
& \leqslant C\left(V^{2}+V\right) \Delta x|\ln \Delta x| .
\end{aligned}
$$

Finally, we bound term IV in (3.8). First observe that

$$
\left\|\int_{t_{j-1}}^{t_{j}} K\left(t_{n}-s\right) * \frac{\partial}{\partial x} f(v(s)) d s\right\|_{\infty} \leqslant \int_{t_{j-1}}^{t_{j}} \frac{C\|f(v(s))\|_{\infty}}{\sqrt{t_{n}-s}} d s \leqslant C V \sqrt{\Delta t}=C V \Delta x
$$

by (3.9). We may therefore integrate from $t_{1}$ to $t_{n-1}$ in the integral in IV. Similarly, the $j=1$ term in the sum of IV is bounded by

$$
\left\|K_{x}\left(t_{n-1}\right)\right\|_{1}\left\|f\left(v_{0}\right)\right\|_{\infty} \Delta t \leqslant C V \Delta t / \sqrt{t_{n}},
$$

and the $j=n$ term is bounded by

$$
\left\|\frac{\partial}{\partial x} f\left(v\left(t_{n-1}\right)\right)\right\|_{\infty} \Delta t \leqslant C V \Delta t / \sqrt{t_{n}} .
$$

These terms may therefore be discarded. Thus, except for these modifications, the sup-norm of the brackets in IV is bounded by

$$
\begin{aligned}
& \left\|\sum_{j=2}^{n-1} K\left(t_{n}-t_{j}\right) * \frac{\partial}{\partial x} f\left(v\left(t_{j-1}\right)\right) \Delta t-\int_{t_{1}}^{t_{n-1}} K\left(t_{n}-s\right) * \frac{\partial}{\partial x} f(v(s-\Delta t)) d s\right\|_{\infty} \\
& +\left\|\int_{t_{1}}^{t_{n-1}} K_{x}\left(t_{n}-s\right) *[f(v(s))-f(v(s-\Delta t))] d s\right\|_{\infty} .
\end{aligned}
$$

The second term in (3.10) is bounded by

$$
\begin{aligned}
C \int_{t_{1}}^{t_{n-1}} \int_{s-\Delta t}^{s}\left\|K_{x}\left(t_{n}-s\right)\right\|_{\infty}\left\|v_{t}(s)\right\|_{1} d s \\
\leqslant C\left(V+V^{2}\right) \int_{t_{1}}^{t_{n-1}} \frac{1}{\left(t_{n}-s\right)^{3 / 2}} \int_{s-\Delta t}^{s} \frac{d \sigma}{\sqrt{\sigma}} d s \\
\leqslant C\left(V+V^{2}\right) \Delta t \int_{t_{1}}^{t_{n-1}} \frac{d s}{\left(t_{n}-s\right)^{3 / 2}(s-\Delta t)^{1 / 2}} \\
\leqslant C\left(V+V^{2}\right) \Delta t^{1 / 2}=C\left(V+V^{2}\right) \Delta x .
\end{aligned}
$$

Now for the first term in (3.10), we use the fact that, for regular functions $w(t)$,

$$
\left|\sum_{j=2}^{n-1} w\left(t_{j}\right) \Delta t-\int_{t_{1}}^{t_{n-1}} w(t) d t\right| \leqslant \Delta t \int_{t_{1}}^{t_{n-1}}\left|w_{t}(t)\right| d t .
$$


Therefore the first term in (3.10) is bounded by

$$
\begin{aligned}
\Delta t \int_{t_{1}}^{t_{n-1}} \| \frac{\partial}{\partial s} & K_{x}\left(t_{n}-s\right) * f(v(s-\Delta t)) \|_{\infty} d s \\
\leqslant & \Delta t \int_{t_{1}}^{t_{n-1}}\left(\left\|K_{x t}\left(t_{n}-s\right)\right\|_{1}\|f(v(s-\Delta t))\|_{\infty}\right. \\
& \left.+\left\|K_{x}\left(t_{n}-s\right)\right\|_{\infty}\left\|f(v(s-\Delta t))_{t}\right\|_{1}\right) d s \\
\leqslant & C \Delta t \int_{t_{1}}^{t_{n-1}}\left[\frac{V}{\left(t_{n}-s\right)^{3 / 2}}+\frac{V+V^{2}}{\left(t_{n}-s\right)^{3 / 2}(s-\Delta t)^{1 / 2}}\right] d s \\
\leqslant & C\left(V+V^{2}\right) \Delta t^{1 / 2}=C\left(V+V^{2}\right) \Delta x
\end{aligned}
$$

The proof is complete.

We are now prepared to derive the final error bound for the approximate solution $u^{n}$ computed from (1.3). We continue to let $v(x, t)$ be the exact solution of (1.1)-(1.2) and $v^{n}=P v\left(\cdot, t_{n}\right)$.

THEOREM 3.3. Assume that $v_{0}$ is of class BV with total variation $V$ and that Assumptions A and B of Section 1 are in force. Then given $T>0$ there is a constant $C$ depending only on $\varepsilon, T$, and $f$ such that the bound

$$
\left|v^{n}-u^{n}\right|_{\infty} \leqslant \frac{C}{\sqrt{t_{n}}}\left[\left|v^{0}-u^{0}\right|_{1}+\left(V+V^{2}\right) \Delta x|\ln \Delta x|\right]
$$

holds for $0<t_{n} \leqslant T$.

Proof. From the representation theorem, Theorem 2.1, applied to the difference equation (1.3), we have that

$$
u^{n}=a^{n} * u^{0}-\sum_{j=1}^{n} a^{n-j} * \frac{\delta}{2 \Delta x} f\left(u^{j-1}\right) \Delta t .
$$

Subtracting this from the definition of $\tau^{n},(3.6)$, we therefore obtain that

$$
\begin{aligned}
v^{n}-u^{n}= & a^{n} *\left(v^{0}-u^{0}\right) \\
& -\sum_{j=1}^{n} \frac{\delta a^{n-j}}{2 \Delta x} *\left[P f\left(v\left(t_{j-1}\right)\right)-f\left(u^{j-1}\right)\right] \Delta t+\tau^{n},
\end{aligned}
$$

where by Lemma 3.2,

$$
\left|\tau^{n}\right|_{\infty} \leqslant C \frac{V+V^{2}}{\sqrt{t_{n}}} \Delta x|\ln \Delta x| \equiv \frac{E}{\sqrt{t_{n}}} .
$$

Now the $j=1$ term in (3.12) is bounded (in the sup-norm) by

$$
\left|\frac{\delta a^{n-1}}{2 \Delta x}\right|_{1}|f|_{\infty} \Delta t \leqslant \frac{E}{\sqrt{t_{n}}}
$$

by Theorem 2.3 , and the $j=n$ term is bounded by

$$
\left(\left\|\frac{\partial}{\partial x} f\left(v\left(t_{j-1}\right)\right)\right\|_{\infty}+\frac{\left|f\left(u^{n-1}\right)\right|_{\infty}}{\Delta x}\right) \Delta t \leqslant \frac{E}{\sqrt{t_{n}}}
$$


by Lemma 3.1. We therefore have from (3.12) that

$$
\left|v^{n}-u^{n}\right|_{\infty} \leqslant C \frac{\left|v^{0}-u^{0}\right|_{1}}{\sqrt{t_{n}}}+\sum_{j=2}^{n-1} \frac{C\left|v^{j-1}-u^{j-1}\right|_{\infty}}{\sqrt{t_{n}-t_{j}}} \Delta t+\frac{2 E}{\sqrt{t_{n}}} .
$$

Multiplying by $\sqrt{t_{n}}$ and letting $E_{n}=\sqrt{t_{n}}\left|v^{n}-u^{n}\right|_{\infty}$, we then obtain

$$
E_{n} \leqslant C\left(\left|v^{0}-u^{0}\right|_{1}+E\right)+C \sqrt{t_{n}} \sum_{j=2}^{n-1} \frac{E_{j-1} \Delta t}{\sqrt{t_{j-1}\left(t_{n}-t_{j}\right)}} .
$$

This is nearly identical in form to (3.4). A discrete version of the proof of (3.1) from (3.4), applied to (3.15), then shows that

$$
E_{n} \leqslant C(T)\left(\left|v^{0}-u^{0}\right|_{1}+E\right),
$$

which is (3.11).

Acknowledgement. We would like to thank the referee for making several suggestions for simplifying the proofs and improving the exposition.

Department of Mathematics

Indiana University

Bloomington, Indiana 47401

Department of Mathematics

University of Michigan

Ann Arbor, Michigan 48109

1. D. HofF, "A finite difference scheme for a system of two conservation laws with artificial viscosity," Math. Comp., v. 33, 1979, pp. 1171-1193.

2. D. HofF, "Invariant regions and finite difference schemes for systems of conservation laws." (To appear.)

3. T. Nishida \& J. Smoller, "A class of convergent finite difference schemes for certain nonlinear parabolic systems," Comm. Pure Appl. Math., v. 36, 1983, pp. 785-808.

4. J. Smoller, Shock Waves and Reaction-Diffusion Equations, Springer-Verlag, New York, 1983.

5. B. Temple, "Systems of conservation laws with invariant submanifolds," Trans. Amer. Math. Soc., v. 280,1983, pp. $781-795$.

6. J. Glimm, "Solutions in the large for nonlinear hyperbolic systems of equations," Comm. Pure Appl. Math., v. 18, 1965, pp. 697-715.

7. Ronald J. DiPerna, "Convergence of the viscosity method for isentropic gas dynamics," Comm. Math. Phys., v. 91, 1983, pp. 1-30. 